\title{
Neighborhood environments and intrinsic capacity interact to affect health related quality of life of older people in New Zealand
}

\begin{abstract}
Objectives:

Following the WHO 2015 policy framework, we tested the effects of older people's intrinsic capacity and their perceptions of their neighborhood environments on mental and physical health related quality of life (QoL) outcomes across two years.

Study design:

Participants (mean age $=66)$ were drawn from two waves of a longitudinal study of aging $(n$ $=2910$ ) in 2016 and 2018. Regression analyses tested the main and interaction effects of intrinsic capacity and neighborhood factors on health related QoL at T2 (controlling for T1). Main outcome measures:

Intrinsic capacity was assessed with number of chronic conditions; Neighborhood perceptions with measures of housing suitability, neighborhood satisfaction, and neighborhood social cohesion; Health related QoL with SF12 physical and mental health component scores. Results:
\end{abstract}

Perceptions of greater neighborhood accessibility and more trust among neighbours were associated with better mental health related QoL two years later, but not to changes in physical health related QoL. A significant interaction between intrinsic capacity and neighborhood access to facilities on physical health related QoL over time showed that those reporting lower neighborhood access experienced a stronger impact of intrinsic capacity on physical health related QoL.

Conclusions: 
The neighborhood environment is important to the wellbeing of older people, and is amenable to policy interventions. At present we need more work focussing on the aspects of the immediate environment that support QoL in older age. This study points to the need for accessible facilities and cohesive neighborhoods to support health.

\section{Keywords}

Neighborhoods; Intrinsic capacity; Mental health; Physical health; Quality of Life; Environment; Healthy Aging 
The World Health Organisation (WHO) 'Framework for Policy for Healthy Aging' [1] has shifted our perspectives on wellbeing for older people. It included recognition of the importance of environments for everyday functioning, providing the impetus for research on the interactions of intrinsic capacity ("the composite of all the physical and mental capacities of an individual" p.28) and environments of aging in predicting quality of life (QoL).

An early explanation of the interaction between environments and intrinsic capacity is provided by the Selection, Optimisation and Compensation (SOC) model [2] which describes how people use psychological, material and social resources to compensate for changes in their physical functioning and maintain their QoL. Baltes [3] held that selection of valued domains of functioning becomes increasingly important in older age; however, this model has not been used to specify relevant environments [4]. A preliminary question is about which specific aspects of environments can support wellbeing in later life.

Environments are conceptualized as more or less proximate to the person from nearby environments of home and neighborhood to more macro policy environments that may compensate for or exacerbate age-related changes in intrinsic capacity. In the present study, we focus on neighborhoods. Awareness of the importance of this aspect of environments has been influenced by the WHO [5] age-friendly model for communities that are supportive, accessible, walkable, and safe. Several authors have since suggested conceptual developments to the model that allow for diversity, reduction of inequalities, or accommodation of older people's changing needs [6] [7] [8] [9]. The theory of personenvironment fit [10] has been influential in ongoing research on the importance of housing and neighborhoods to wellbeing [11]. From this perspective, if personal needs are not supported by the resources offered by the environment, then the QoL of older people is affected [7]. In general, research has demonstrated that older people are more vulnerable to the consequences of poor housing and neighborhood conditions due to reduced mobility, 
spending greater proportions of time at home, and having higher rates of disability and health-related conditions [12], while neighborhood facilities are positively related to health and functioning [13] [14] and QoL [9]. However, such research is limited; despite early environmental theorising, Wahl et al [4] have noted that the role of the immediate physical, spatial, and technical environment has largely been neglected in gerontological research. In general, there is evidence that neighborhoods are important to older people [15] although their links to wellbeing are under researched [16].

The SOC model [3] also leads us to specify the changes in intrinsic capacity that require support. The International Consortium on Metrics and Evidence for Healthy Aging argues that ongoing work to support the WHO [1] strategy in action must include scientific evidence for the aspects of intrinsic capacity in older people that should be globally assessed [17]. In this study, we focus on chronic health conditions as an indicator of intrinsic capacity, i.e., medical diagnoses of disease, disorder or injury which are included in the WHO International Classification of Functioning, Disability and Health [18]. Beard and colleagues [19] summarised objective estimates of physical and cognitive functioning among older adults to provide an assessment of intrinsic capacity. Using this assessment, they found that multiple chronic conditions was a significant correlate of intrinsic capacity scores.

We tested a model of the relationships between older people's number of chronic health conditions (as a proxy for level of intrinsic capacity), and their perceptions of their housing and neighborhood environments (housing satisfaction, neighborhood accessibility, neighborhood safety, and social cohesion). These variables were expected to predict mental and physical health related QoL outcomes across two years. We also expected an interaction between intrinsic capacity and neighborhood environments in predicting mental and physical health outcomes. Our predictions are: 
1. Intrinsic Capacity and Perceptions of Environments will be positively associated with changes in Mental and Physical Health related QoL over two years.

2. There will be an interaction between Intrinsic Capacity and Perceptions of Environments in the prediction of Mental and Physical Health such that the positive relationship of Intrinsic Capacity with Mental and Physical Health related QoL over two years will be stronger when Perceptions of Environments are more positive.

\section{Method}

Data were drawn from responses to the 2016 and 2018 Health, Work and Retirement longitudinal surveys. [20] Included participants were adults aged 55+ who were randomly selected from the New Zealand electoral roll and responded to the 2016 survey $(n=4029)$, provided complete data on demographic control variables, number of chronic conditions (as an indicator of level of intrinsic capacity), environmental perceptions and physical and mental health $(n=3666)$, and provided complete data on indicators of physical and mental health at follow-up $(n=2910)$.

\section{Measures}

Self-reported age, gender and living standards were included as demographic control variables. Living standards was assessed using the Economic Living Standards Index short form (ELSI-SF), a 25-item non-income measure of material wealth [21]. Scores on the ELSISF range from 0-31, with higher scores indicating better living standards.

\subsection{Environments}

Environments were assessed by indicators of housing suitability ( 8 items, example item: 'My home meets all my needs'), neighborhood satisfaction ('I am satisfied with my neighborhood'), and access to facilities in neighborhood (3 items: 'I am close enough to important facilities'), on a scale of 1 'no, definitely not' to 5 'yes, definitely', adapted from Heywood et al., [22] and Oswald et al., [23]. Neighborhood trust was assessed using the trust 
subscale of the Neighborhood Social Cohesion tool [24] (6 items: 'People in this area would do something if a house was being broken into') on a scale of 1 'strongly disagree' to 5

'strongly agree'. Total scores for each environment factor were calculated as mean component item ratings (score ranges 1-5). Component items and scale reliability statistics are presented in Appendix 1.

\subsection{Intrinsic Capacity}

Participants reported whether a health professional had ever told them that they had any of the following fourteen conditions: Arthritis or rheumatism; Disorders of the neck or back; Diabetes; Disability; Heart trouble (e.g., angina or heart attack); High blood pressure or hypertension; Depression; Other mental illness; Respiratory condition (e.g., bronchitis, asthma); Sleep disorder; Stroke; Active or chronic gout; Active/chronic hepatitis, cirrhosis or other liver condition; Cancer. A total score ranging from 0-14 was calculated with higher scores indicating fewer diagnoses and therefore higher intrinsic capacity.

\subsection{Mental and Physical Health Related Quality of Life}

Physical and mental health QoL were assessed using items of the SF-12 v2 Australian and New Zealand form. [25] Standardized totals for the physical and mental health component scores were calculated with reference to normative subscale scores and factor coefficients for the New Zealand population [26] to facilitate interpretation relative to an adult population mean score of 50 with a standard deviation of 10 .

\section{Analysis}

Data analyses were completed using Mplus v8.3. Maximum likelihood estimation with robust standard errors (MLR) was used for all analyses. Hierarchical regression analyses were used to assess the main effects of (mean centered) Intrinsic Capacity (IC) and Environmental factors and their interactions in the prediction of changes in physical health $(\mathrm{PH})$ and mental health $(\mathrm{MH})$ over time. In step 1, the impacts of baseline $\left(\mathrm{T}_{1}\right) \mathrm{PH}$, age, 
gender, and material living standards on $\mathrm{PH}$ and $\mathrm{MH}$ at follow up $\left(\mathrm{T}_{2}\right)$ were estimated. In step 2, the main effects of IC and each Environmental factor were additionally estimated. In step 3, interactions of IC and each Environment factor were estimated. Unstandardized beta and associated $95 \%$ confidence intervals are reported, along with the variance explained $\left(\mathrm{R}^{2}\right)$ in each model step. An alpha of .05 was used for all tests of significance.

\section{Results}

\section{Participant group description}

Correlations among study variables, means and standard deviations are reported in Table 1.

\section{Physical Health Related QoL}

Table 2 reports the results of the three-step hierarchical regression of $\mathrm{PH}$ on IC and environmental factors, and their interaction. At step 1, baseline $\mathrm{PH}$, age, gender, and material living standards explained 51\% of the variance in $\mathrm{PH}$ at follow-up. Higher baseline PH, lower age, female gender, and higher living standards were associated with higher $\mathrm{PH}$ at follow up. Step 2 explained an additional $1.3 \%$ of variance, with IC positively associated with PH over time. Environmental factors were not associated with PH at follow up. In step 3, the interaction term for neighborhood access to facilities and IC was significant (additional $0.1 \%$ variance in $\mathrm{PH}$ over time). Tests of simple slopes (Figure 1) indicate the positive association between IC and PH over time was stronger when neighborhood access to facilities was low (1SD: $B=0.92, t=7.27, p<.001)$ relative to high $(+1 \mathrm{SD}: B=0.57, t=4.28, p<.001)$.

\section{Mental Health Related QoL}

The right-hand columns of Table 2 report the results of the three-step hierarchical regression of mental health $(\mathrm{MH})$ on IC, environmental factors, and their interaction. At step 1, baseline $\mathrm{MH}$, and material living standards explained $40.6 \%$ of the variance. Higher baseline $\mathrm{MH}$ and higher living standards were associated with greater $\mathrm{MH}$ at follow up. Step 
2 explained an additional $1.4 \%$ of the variance, with greater IC, greater neighborhood accessibility and greater neighborhood trust associated with greater $\mathrm{MH}$ at follow up. Step 3 indicated no significant moderating effects.

\section{Discussion}

As predicted, intrinsic capacity was positively related to changes in physical and mental health related QoL over two years. Regarding the immediate environment, perceptions of greater neighborhood accessibility and more trust among neighbours was associated with better mental health related QoL two years later (but not to changes in physical health related QoL). However, there was a significant interaction between the effects of intrinsic capacity and neighborhood access to facilities on physical health QoL over time: indicating that the impact of low intrinsic capacity on physical health related QoL over time was greater for those with less access to facilities. Because health related QoL at time 1 strongly predicts

QoL at time 2, the additional variance explained by other variables is small. By explaining this additional variance in QoL over time, these findings highlight aspects of the neighborhood environment that have important implications for wellbeing among older community dwellers.

The importance of neighborhood accessibility and social cohesion among neighbours is not surprising. The WHO age-friendly model [5] emphasised the importance of communities that are supportive, accessible, walkable, and safe. Previous work has highlighted the ways in which informal relationships and every day interactions in neighborhoods enhance the well-being of older adults aging in place [27] and social cohesion has been identified as a key aspect of the neighborhood social environment. [28] Research has shown that access to neighborhood facilities is positively related to QoL [9] [13] [14] and the present study emphasises the importance of these aspects of the environment to changes in health related QoL over time. 
The importance of accessibility is emphasised by these findings. For those with limited intrinsic capacity (manifested in diagnosed conditions) lack of accessibility to important facilities is most likely to negatively affect physical functioning and health related QoL. This study only asked for perceptions of general accessibility and future work should more closely examine which facilities are valued by older people such as community recreational facilities, community services, and public transportation. [29]

It is important to recognise the role of socioeconomic standards (SES) in health and communities. Lower living standards were strongly correlated with health related QoL in this study, and lower SES has also been linked to deprived neighborhoods and social exclusion, both from neighbours and the wider community [30]. Future research on environments of aging must take account of the effects of aspects of the broader social environment (such as SES) on the immediate environment of people aging in communities.

The main limitation of this study is the assessment of intrinsic capacity. Number of chronic diagnoses is a proxy that indicates limitations in functioning ability and has been shown to correlate with lower intrinsic capacity [19]. Further work could be done to refine this type of measure by considering which chronic diseases are more likely to reflect poorer capacity. However, this would remain an indication only and as we develop a globally recognised assessment tool [19] more direct measures of physical and cognitive capacity can be used.

\section{Conclusions}

The immediate environment including neighborhoods is an important contributor to the wellbeing of older people, and an aspect that is amenable to policy intervention by state and local authorities. At present we need more work focussing on the important aspects of the environment that support QoL in older age. This study points to the need for accessible 
facilities and cohesive neighborhoods to support health, which suggests at least two sites for further study and potential intervention. 


\section{Funding}

This work was supported by the New Zealand Ministry of Business Innovation and Employment Endeavour Fund programme [grant number MAUX1705].

\section{References}

1. World Health Organisation, World Report on Aging and Health. 2015.

2. Baltes, P.B. and M.M. Baltes, Psychological perspectives on successful aging: The model of selective optimization with compensation, in Successful aging: Perspectives from the behavioral sciences, P.B. Baltes and M.M. Baltes, Editors. 1990, Cambridge University Press: New York. p. 1-34.

3. Baltes, P.B., On the incomplete architecture of human ontogeny: Selection, optimization, and compensation as foundation of developmental theory. American Psychologist, 1997. 52: p. 366-380.

4. Wahl, H.-W., S. Iwarsson, and F. Oswald, Aging well and the environment: Toward an integrative model and research agenda for the future. The Gerontologist, 2012. 52(3): p. 306316.

5. World Health Organization, Checklist of essential features of age-friendly cities. 2007, Geneva: WHO.

6. Buffel, T., C. Phillipson, and T. Scharf, Aging in urban environments: Developing 'agefriendly' cities. Critical Social Policy, 2012. 32(4): p. 597-617.

7. Keating, N., J. Eales, and J.E. Phillips, Age-friendly rural communities: Conceptualizing 'best-fit'. Canadian Journal on Aging/La Revue canadienne du vieillissement, 2013. 32(04): p. 319-332.

8. Shank, K.S.H. and M.P. Cutchin, Processes of developing 'community livability'in older age. Journal of Aging Studies, 2016. 39: p. 66-72.

9. Stephens, C., et al., Livable Environments and the Quality of Life of Older People: An Ecological Perspective. The Gerontologist, 2019. 39(4): p. 675-685. 
10. Peace, S., et al., Environment and aging, in Aging in society, J. Bond, et al., Editors. 2007, Sage: UK. p. 209-234.

11. Peace, S., C. Holland, and L. Kellaher, 'Option recognition' in later life: variations in aging in place. Aging and Society, 2011. 31(05): p. 734-757.

12. Costa-Font, J., O. Mascarilla-Miro', and D. Elvira, Aging in place? Exploring elderly people's housing preferences in Spain. Urban Studies, 2009. 46(2): p. 295-316.

13. Bowling, A., et al., Do perceptions of neighborhood environment influence health? Baseline findings from a British survey of aging. J Epidemiol Community Health, 2006. 60(6): p. 476483.

14. Byles, J.E., et al., Supporting housing and neighborhoods for healthy aging: Findings from the H ousing and I ndependent L iving S tudy (HAIL). Australasian Journal on Aging, 2014. 33(1): p. 29-35.

15. van der Pas, S., et al., Features of home and neighborhood and the liveability of older South Africans. European journal of aging, 2015. 12(3): p. 215-227.

16. Sixsmith, J., et al., Healthy aging and home: The perspectives of very old people in five European countries. Social Science \& Medicine, 2014. 106: p. 1-9.

17. Ritu Sadana, Anshu Banerjee, and on behalf of the WHO Consortium on Metrics and Evidence for Healthy Aging, Metrics and evidence for healthy aging. Bulletin of the World Health Organization, 2019. 97: p. 792-792A.

18. Üstün, T.B., et al., The International Classification of Functioning, Disability and Health: a new tool for understanding disability and health. Disability and rehabilitation, 2003. 25(1112): p. 565-571.

19. Beard, J.R., et al., The structure and predictive value of intrinsic capacity in a longitudinal study of aging. BMJ open, 2019.9(11).

20. Allen J, Alpass F M, and Stephens C, New Zealand Health, Work and Retirement Longitudinal Study, in Encyclopedia of Gerontology and Population Aging, Gu D and Dupre M E, Editors. 2019, Springer International Publishing: Cham. p. 1-7. 
21. Jensen, J., M. Spittal, and V. Krishnan, ELSI Short Form. User Manual for a direct measure of living standards. 2005, Wellington, NZ: New Zealand Ministry of Social Development.

22. Heywood, F., C. Oldman, and R. Means, Housing and Home in Later Life. 2002, Milton Keynes: Open University Press.

23. Oswald, F., et al., Homeward bound: Introducing a four-domain model of perceived housing in very old age. Journal of Environmental Psychology, 2006. 26(3): p. 187-201.

24. Stafford, M., et al., Measuring the social environment: social cohesion and material deprivation in English and Scottish neighborhoods. Environment and Planning A, 2003. 35(8): p. 1459-1475.

25. Ware, J.E., et al., SF-12v2 ${ }^{\mathrm{TM}}$ : How to score version 2 of the $S F-12 \AA$ health survey. 2002.

26. Frieling, M.A., W.R. Davis, and G. Chiang, The SF-36v2 and SF-12v2 health surveys in New Zealand: norms, scoring coefficients and cross-country comparisons. Australian and New Zealand journal of public health, 2013. 37(1): p. 24-31.

27. Gardner, P.J., Natural neighborhood networks-Important social networks in the lives of older adults aging in place. Journal of aging studies, 2011. 25(3): p. 263-271.

28. Cramm, J.M. and A.P. Nieboer, Social cohesion and belonging predict the well-being of community-dwelling older people. BMC Geriatrics, 2015. 15(1): p. 30.

29. Cho, J., C. Cook, and M.J. Bruin, Functional ability, neighborhood resources and housing satisfaction among older adults in the US. Journal of Housing for the Elderly, 2012. 26(4): p. $395-412$.

30. Prattley, J., et al., Area effects on the level and development of social exclusion in later life. Social Science \& Medicine, 2020. 246: p. 112722. 
Table 1. Bivariate correlation, and descriptive indicators for model variables $(n=2910)$.

\begin{tabular}{|c|c|c|c|c|c|c|c|c|c|c|c|c|c|}
\hline & 1 & 2 & 3 & 4 & 5 & 6 & 7 & 8 & 9 & 10 & 11 & $\mathrm{M}$ & SD \\
\hline 1. Age & & & & & & & & & & & & 65.77 & 6.46 \\
\hline 2. Female & -0.06 & & & & & & & & & & & 0.56 & 0.50 \\
\hline 3. Economic living standard & 0.08 & $-0.05 *$ & & & & & & & & & & 24.86 & 5.90 \\
\hline 4. Intrinsic capacity & -0.13 & $-0.02 \mathrm{~ns}$ & 0.33 & & & & & & & & & 12.04 & 1.70 \\
\hline 5. Housing satisfaction & 0.10 & $0.01^{\mathrm{ns}}$ & 0.42 & 0.19 & & & & & & & & 4.46 & 0.74 \\
\hline 6. Neighborhood satisfaction & 0.07 & $0.01^{\mathrm{ns}}$ & 0.26 & 0.12 & 0.65 & & & & & & & 4.51 & 0.89 \\
\hline 7. Neighborhood access to facilities & 0.08 & $0.03^{\mathrm{ns}}$ & 0.30 & 0.12 & 0.45 & 0.31 & & & & & & 4.58 & 0.65 \\
\hline 8. Neighborhood trust & 0.07 & $-0.02^{\mathrm{ns}}$ & 0.35 & 0.16 & 0.34 & 0.38 & 0.31 & & & & & 4.14 & 0.64 \\
\hline 9. Physical Health $\mathrm{T}_{1}$ & -0.19 & $0.00^{\mathrm{ns}}$ & 0.35 & 0.52 & 0.17 & 0.09 & 0.13 & 0.15 & & & & 47.29 & 9.77 \\
\hline 10. Physical Health $T_{2}$ & -0.21 & $0.03^{\text {ns }}$ & 0.34 & 0.47 & 0.18 & 0.10 & 0.13 & 0.14 & 0.70 & & & 46.07 & 10.39 \\
\hline 11. Mental Health $\mathrm{T}_{1}$ & 0.07 & $-0.02^{\mathrm{ns}}$ & 0.42 & 0.32 & 0.32 & 0.20 & 0.26 & 0.28 & 0.17 & 0.23 & & 50.93 & 9.23 \\
\hline 12. Mental Health $\mathrm{T}_{2}$ & $0.04^{*}$ & $-0.03^{\mathrm{ns}}$ & 0.42 & 0.32 & 0.29 & 0.19 & 0.25 & 0.28 & 0.27 & 0.24 & 0.61 & 50.16 & 9.85 \\
\hline
\end{tabular}

Note. All $p<.001$ unless otherwise indicated; ${ }^{n s} p>.05 ; * p<.05$. 
Table 2. Hierarchical regression of Physical and Mental Health on intrinsic capacity, environments and their interaction $(n=2910)$.

\begin{tabular}{|c|c|c|c|c|c|c|c|c|c|c|}
\hline & \multicolumn{5}{|c|}{ Physical Health $\left(T_{2}\right)$} & \multicolumn{5}{|c|}{ Mental Health $\left(T_{2}\right)$} \\
\hline & $R^{2}$ & $B$ & $95 \% C I$ & $\mathrm{t}$ & $\mathrm{p}$ & $R^{2}$ & $B$ & $95 \% C I$ & $\mathrm{t}$ & $\mathrm{p}$ \\
\hline Step 1 & 0.510 & & & & & 0.406 & & & & \\
\hline Health QoL domain $\left(\mathrm{T}_{1}\right)$ & & 0.68 & $(0.65,0.72)$ & 37.63 & 0.000 & & 0.56 & $(0.52,0.60)$ & 26.94 & 0.000 \\
\hline Age & & -0.16 & $(-0.21,-0.12)$ & -7.30 & 0.000 & & -0.02 & $(-0.07,0.02)$ & -0.99 & 0.322 \\
\hline Female gender & & 0.62 & $(0.09,1.15)$ & 2.29 & 0.022 & & -0.23 & $(-0.79,0.32)$ & -0.82 & 0.410 \\
\hline Economic living standards & & 0.21 & $(0.16,0.27)$ & 7.69 & 0.000 & & 0.34 & $(0.28,0.39)$ & 10.40 & 0.000 \\
\hline Step 2 & 0.523 & & & & & 0.420 & & & & \\
\hline Intrinsic capacity (IC) & & 0.76 & $(0.56,0.95)$ & 7.67 & 0.000 & & 0.56 & $(0.35,0.76)$ & 5.35 & 0.000 \\
\hline Housing satisfaction & & 0.50 & $(-0.03,1.03)$ & 1.87 & 0.062 & & 0.21 & $(-0.41,0.83)$ & 0.70 & 0.504 \\
\hline Neighborhood satisfaction & & -0.12 & $(-0.53,0.29)$ & -0.59 & 0.553 & & -0.07 & $(-0.54,0.40)$ & -0.31 & 0.760 \\
\hline Neighborhood access to facilities & & 0.24 & $(-0.22,0.70)$ & 1.02 & 0.307 & & 0.67 & $(0.12,1.22)$ & 2.38 & 0.017 \\
\hline Neighborhood trust & & -0.12 & $(-0.61,0.36)$ & -0.49 & 0.622 & & 0.91 & $(0.41,1.41)$ & 3.53 & 0.000 \\
\hline Step 3 & 0.524 & & & & & 0.421 & & & & \\
\hline IC*Housing satisfaction & & -0.11 & $(-0.38,0.16)$ & -0.93 & 0.354 & & 0.04 & $(-0.32,0.40)$ & 0.20 & 0.842 \\
\hline IC*Neighborhood satisfaction & & 0.06 & $(-0.16,0.27)$ & 0.56 & 0.574 & & 0.05 & $(-0.23,0.33)$ & 0.32 & 0.748 \\
\hline IC*Neighborhood access to facilities & & -0.27 & $(-0.53,-0.01)$ & -2.20 & 0.028 & & 0.12 & $(-0.24,0.33)$ & 0.65 & 0.517 \\
\hline IC*Neighborhood trust & & 0.22 & $(-0.03,0.47)$ & 1.79 & 0.073 & & -0.17 & $(-0.47,0.13)$ & -1.11 & 0.265 \\
\hline
\end{tabular}

Note: $B=$ unstandardized beta coefficient; $\mathrm{T}_{1}$ Health related QoL domain at 2016 survey; $\mathrm{T}_{2}$ Health related QoL domain at 2018 survey; PH model intercept b0 = 22.924; MH model intercept $\mathrm{b} 0=13.34$. 
Figure 1. Impact of IC on physical health related QoL over time in neighborhoods perceived to have low $v s$. high access to facilities. 


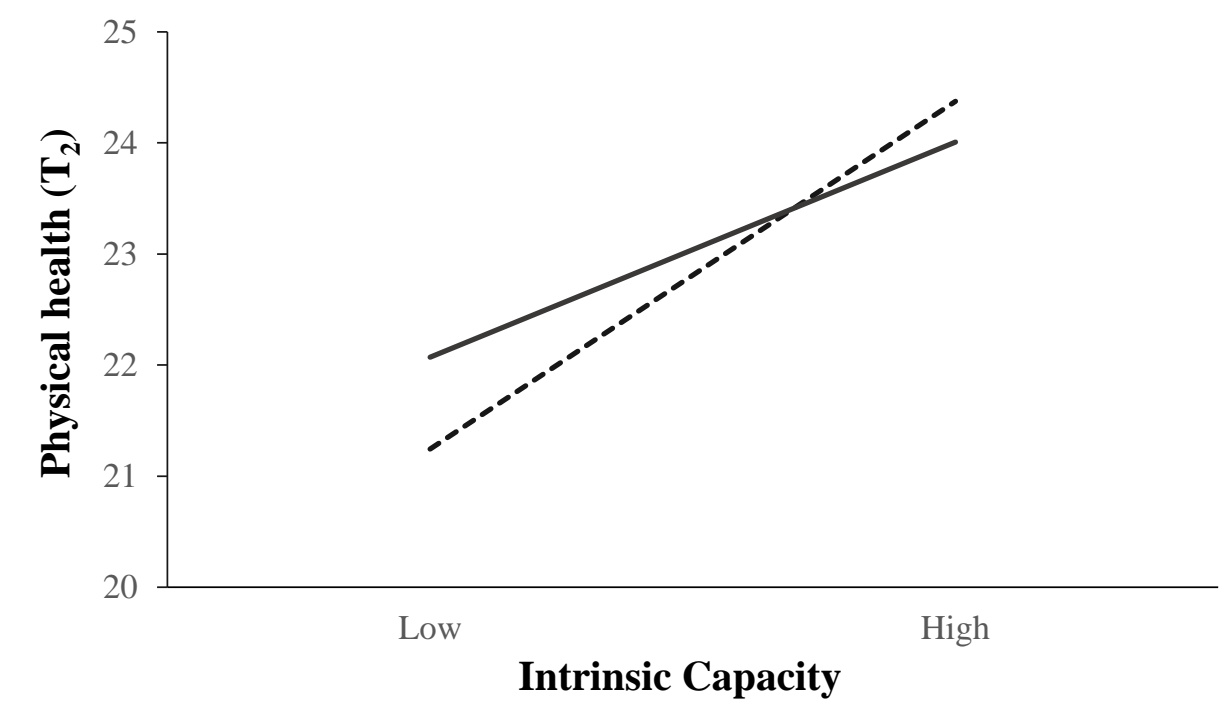

----- Low access to facilities — High access to facilities 
